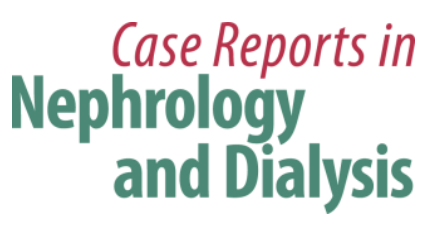

\title{
Drug-Immune Thrombocytopenia with Thrombosis versus Heparin-Induced Thrombocytopenia: A Critical Clinical Controversy
}

\author{
Hassan Al-Jafar ${ }^{a} \quad$ Anas Al-Yousef ${ }^{b} \quad$ Somaya Al-Shatti ${ }^{\mathrm{C}}$ \\ Khalifa Al-Banwan ${ }^{\text {d }}$ \\ ${ }^{a}$ Hematology Department and ${ }^{b}$ Internal Medicine and Nephrology, AMIRI Hospital, \\ 'Platelet Serology Laboratory, Kuwait Central Blood Bank, and ${ }^{\mathrm{d}}$ Microbiology Department, \\ AMIRI Hospital, Kuwait City, Kuwait
}

\section{Key Words}

Drug-immune thrombocytopenia - Heparin-induced thrombocytopenia - Vancomycin . Phlegmasia cerulea dolens · Thrombosis · Controversy

\begin{abstract}
Heparin-induced thrombocytopenia (HIT) is a type of drug-induced immune thrombocytopenia (DITP). DITP is a rare and challenging clinical issue, especially when it is associated with thrombosis. A 62-year-old woman was admitted to our institution with end-stage renal failure. She received heparin for hemodialysis. Six days later, she became febrile and was treated with vancomycin and amikacin antibiotics. Two days after starting the vancomycin, she developed severe thrombocytopenia with extensive gangrenous deep vein thrombosis in her right leg, which required a below-the-knee amputation. The HIT test yielded positive results when heparin was already stopped, but her platelet count did not regenerate even after 3 months of heparin-free treatment. Courses of vancomycin treatment were given during several febrile episodes over the long period of severe thrombocytopenia. The patient was given both anti-immune thrombocytopenia and anticoagulant treatments because of both severe persistent thrombocytopenia and recurrent thrombotic episodes. The patient died as a result of severe thrombocytopenia, recurrent infection, and blood loss from the amputation site. Vancomycin is known to cause DITP, thrombosis, and immune complexes. DITP is a bleeding disorder, whereas HIT is a controversial thrombotic disorder. HIT tests can be influenced by cross-reacting antibodies and many other factors. Thus, there is no single method that can
\end{abstract}

\begin{tabular}{|c|c|}
\hline KARGER 125 & $\begin{array}{l}\text { Hassan A. Al-Jafar } \\
\text { Hematology Department } \\
\text { AMIRI Hospital } \\
\text { Kuwait City (Kuwait) } \\
\text { E-Mail cbc9 chotmail.com }\end{array}$ \\
\hline
\end{tabular}




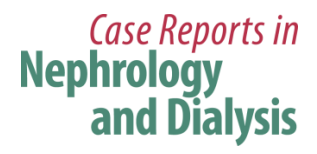

\begin{tabular}{l|l}
\hline Case Rep Nephrol Dial 2015;5:152-159 \\
\hline DOI: 10.1159/000435806 & $\begin{array}{l}\text { C 2015 S. Karger AG, Basel } \\
\text { www.karger.com/cnd }\end{array}$ \\
\hline
\end{tabular}

Al-Jafar et al.: Drug-Immune Thrombocytopenia with Thrombosis versus Heparin-Induced Thrombocytopenia: A Critical Clinical Controversy

be considered $100 \%$ effective in confirming the HIT diagnosis. Anticoagulants must be used with great caution in patients with suspected DITP. Treatment of HIT-positive cases requires both clinical correlation and experience rather than reliance on HIT tests alone.

(C) 2015 S. Karger AG, Basel

\section{Introduction}

Thrombocytopenia is a common hematological abnormality, especially in hospitalized patients. Many factors contribute to the development of a low platelet count, including infections, hemodilution, and drug treatments; consequently, determining the cause of this condition can be difficult. Drugs may cause thrombocytopenia through a variety of mechanisms, including myelosuppression, peripheral destruction, and immune reactions. Immune thrombocytopenia (ITP) induced by drugs is a unique syndrome known as drug-induced immune thrombocytopenia (DITP), caused by drug-dependent antibodies (DDAbs) that bind to platelets. DITP is typically severe and may result in life-threatening bleeding and/or thrombosis, as could occur in vancomycin-induced ITP given that vancomycin is defined as a thrombotic drug $[1,2]$. There are two types of ITP. The primary ITP type is an acquired immunemediated disorder characterized by isolated thrombocytopenia and defined by a peripheral blood platelet count below $100 \times 10^{9} / \mathrm{l}$ and the absence of any obvious initiating and/or underlying cause of the thrombocytopenia [3]. The term secondary ITP type has been proposed to broadly include all forms of immune-mediated thrombocytopenia except primary ITP [4].

Heparin-induced thrombocytopenia (HIT) is a type of DITP defined as a decrease in the platelet count during or shortly following exposure to heparin. The central feature of HIT is thrombocytopenia caused by antibody-mediated attacks directed against neoepitopes on a 'self' protein - platelet factor 4 (PF4) - that are expressed when PF4 is bound to heparin or certain other polyanions. Platelet activation of PF4 can be viewed as an acquired hypercoagulability disorder, with increased thrombin generation in vivo and an increased risk of arterial and venous thrombosis [5]. There are two types of HIT. Type 1 is not immune-mediated or associated with thromboembolic or hemorrhagic events and usually presents as a mild, transient decrease in platelets occurring 1-2 days after exposure to heparin or its derivatives. The platelet count usually does not drop below $100 \times 10^{9} / \mathrm{l}$ and generally recovers spontaneously even with continued exposure to these medications [6]. This condition is also known as heparin-associated thrombocytopenia, a nonimmunological response to heparin treatment mediated by a direct interaction between heparin and circulating platelets that causes platelet clumping or sequestration [7]. Type 2 is a prothrombotic and potentially lethal disorder caused by platelet, endothelial, and monocyte-activating antibodies that target multimolecular complexes of PF4 and heparin [8]. Diagnosis of HIT type 2 is based on the clinical symptoms of thrombocytopenia and the results of an immunoassay for PF4/heparin complex antibodies. It remains unclear why some patients develop HIT. The platelet counts do not return to normal levels unless heparin administration is stopped, although the platelet count usually rises to above normal levels in 5-7 days. In contrast, in ITP, the thrombocytopenia persists without commencing immunesuppressive or thrombopoietic drugs [9].

ITP is primarily a bleeding disorder, but it is paradoxically also associated with venous thromboembolism. A survey of the UK General Practice Research Database, which included 1,070 adults with primary ITP over 15 years, demonstrated an increased risk for venous thromboembolic events in patients with primary ITP [10]. Aledort et al. reported 18 thrombotic episodes, including 5 arterial events, in 10 of 186 patients with ITP. Several mecha- 


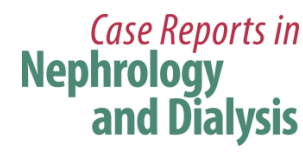

\begin{tabular}{l|l}
\hline Case Rep Nephrol Dial 2015;5:152-159 \\
\hline DOI: 10.1159/000435806 & $\begin{array}{l}\text { C 2015 S. Karger AG, Basel } \\
\text { www.karger.com/cnd }\end{array}$ \\
\hline
\end{tabular}

Al-Jafar et al.: Drug-Immune Thrombocytopenia with Thrombosis versus Heparin-Induced Thrombocytopenia: A Critical Clinical Controversy

nisms have been proposed to explain the thrombotic tendency observed with ITP, including circulating platelet-leukocyte-monocyte aggregates, endothelium-activating antibodies, large proportions of young activated platelets, and platelet microparticles released during immune platelet destruction [11]. The platelet microparticles, while serving the physiological purpose of protecting against bleeding in thrombocytopenia, may also promote thrombosis. Activation of the complement system was also shown to contribute to the thrombocytopenia in ITP either by enhancing clearance of opsonized platelets or by direct damage. Many drugs are also known to cause iatrogenic thrombosis, including vancomycin, lenalidomide, carboplatin, clopidogrel, cisplatin, conjugated estrogens, contraceptives, cyclosporine, estramustine, combined estrogens and progestins, gemcitabine, ketorolac, mitomycin-C, quinine tacrolimus, and valacyclovir [12].

The case presented here illustrates the potential confusion between HIT and DITP, especially in conjunction with thrombosis, with respect to the clinical picture, laboratory results, and treatment methods. This phenomenon occurs in clinical practice, mainly in nephrology wards where both heparin for dialysis and vancomycin for infection treatment are commonly used. It is known that both drugs can cause thrombocytopenia and thrombosis. To our knowledge, it is the first case report in the literature discussing the diagnostic challenge and the clinical controversy between HIT associated with thrombosis and DITP associated with thrombosis; both are rare and serious events with a need to differentiate their diagnosis and management approaches.

\section{Case Presentation}

A 62-year-old woman who provided written and signed consent to publish this case report was admitted to our institution with a history of diabetes mellitus type II, hypertension, and chronic kidney disease secondary to diabetic nephropathy and a hysterectomy 15 years earlier. She presented with weakness, malaise, and loss of appetite. Complete blood count analysis revealed the following: white blood cell count $10.1 \times 10^{9} /$; hemoglobin level 113 $\mathrm{g} / \mathrm{l}$; mean corpuscular volume $84 \mathrm{fl}$; mean corpuscular hemoglobin level $31 \mathrm{pg} / \mathrm{cell}$, and platelet count 264. Further blood tests revealed the following: glucose level $7.8 \mathrm{~mol} / \mathrm{l}$; urea nitrogen level $28.1 \mathrm{mg} / \mathrm{dl}$; creatinine $877 \mathrm{~mol} / \mathrm{l}$; sodium $134 \mathrm{~mol} / \mathrm{l}$, and potassium $3.5 \mathrm{~mol} / \mathrm{l}$. Four days after admission, the patient underwent hemodialysis with unfractionated heparin. Six days after the administration of heparin, she became febrile and was treated with the antibiotics vancomycin and amikacin. That was on day 6 after holding the heparin, which was promptly day 2 after vancomycin treatment had been initiated. Two days after commencing the vancomycin, her platelet count started to drop gradually, decreasing to $21 \times$ $10^{9} /$ l over 8 days, which was day 16 since heparin was held. The HIT test was ordered by the nephrologists. Thereafter, a hematologist was consulted for the persistent thrombocytopenia, as heparin had already been stopped 16 days ago. Because her platelet count slightly regenerated (from 21 to $25 \times 10^{9} / \mathrm{l}$ ), no additional steps were taken other than avoiding heparin, as the patient was scheduled to undergo permacath insertion for hemodialysis the next day. The following day, the platelet count dropped to $19 \times 10^{9} / \mathrm{l}$. The patient was treated with steroids to increase the platelet level for the permacath insertion. Both platelet transfusion (owing to the possible HIT diagnosis) and heparinoid anticoagulants (owing to the urgency of the permacath insertion surgery) were avoided.

Because her platelet count continued to drop to $10 \times 10^{9} / \mathrm{l}$ over the next $24 \mathrm{~h}$, methylprednisolone was given while waiting for the HIT test result. Because the platelet drop occurred following the fever and the vancomycin administration, a secondary ITP that 


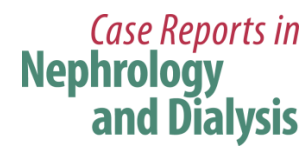

\begin{tabular}{l|l}
\hline \multicolumn{2}{l}{ Case Rep Nephrol Dial 2015;5:152-159 } \\
\hline DOI: 10.1159/000435806 & $\begin{array}{l}\text { C 2015 S. Karger AG, Basel } \\
\text { www.karger.com/cnd }\end{array}$ \\
\hline
\end{tabular}

Al-Jafar et al.: Drug-Immune Thrombocytopenia with Thrombosis versus Heparin-Induced Thrombocytopenia: A Critical Clinical Controversy

could have been caused by DITP was considered. The next day, the patient developed a swollen, hot, and tender right leg. Doppler ultrasound revealed extensive progressive lower limb deep vein thrombosis. An inferior vena cava filter was inserted on the same day. The HIT test result was revealed positive result. Her platelet count rose to $23 \times 10^{9} / \mathrm{l}$ following the administration of methylprednisolone. She was labeled a HIT case, and the pretest probability 4Ts score was an intermediate score.

Bivalirudin (Angiomax) was started, but the deep vein thrombosis advanced to acute phlegmasia cerulea dolens requiring above-the-knee amputation. The leg had become gangrenous with continuous blood oozing caused by both the low platelet count and the anticoagulant drugs. Hemoglobin levels had also dropped to $8.6 \mathrm{~g} / \mathrm{dl}$ because of the continuous bleeding, requiring the transfusion of 2 units of blood. Intravenous immunoglobulin (IVIG) was commenced to treat the DITP, and the platelet count started to increase. On the fourth day of IVIG treatment, the patient developed right thalamic lacunar infarction. The IVIG treatment was continued for 4 weeks along with bivalirudin. The platelet count responded well to the IVIG treatment, reaching $98 \times 10^{9} / \mathrm{l}$ after 6 days but dropping to $26 \times 10^{9} / \mathrm{l}$ over 4 weeks. The amputation site started to bleed again and developed an infection that required debridement. During her long-term admission, the patient received four different courses of vancomycin on days $13,16,34$, and 75 , at doses of 500-1,000 mg, along with $240 \mathrm{mg}$ of amikacin, based on the findings from the blood and wound cultures. The patient was also treated with other antibiotics during the course of treatment, including ceftriaxone on day 26, marine, and linezolid on day 52. Romiplostim, which was the third and a U.S. Food and Drug Administration-approved ITP treatment, was administered promptly when IVIG was stopped. In response to romiplostim treatment, the platelet count increased promptly to the normal values, and the bleeding stopped. The patient, however, developed a third thrombosis in her right iliac vein. The next dose of romiplostim was administered on time, 1 week later, when the patient had been hematologically stabilized, i.e., no more bleeding occurred and a normal cell count was achieved. Bivalirudin was replaced by warfarin because of the normalized platelet count as well as the recurrent thrombosis events and the need for hemodialysis. The patient developed septicemia from the amputation site, which caused delayed healing and infection. The wound site was debrided several times, but no improvement was noted. The amputation site showed positive results on several swab cultures, but antibiotic treatment was ineffective. The patient was kept on conservative management strategies until her death after 3 months. Although vancomycin was suspected as the cause of her thrombocytopenia, no vancomycin antibody test was available in our laboratory to confirm the findings in this patient. No malignancy screening tests were conducted because she was not suspected to have a malignancy, and no postmortem autopsy was performed because it was considered unacceptable based on religious factors in her community.

\section{Discussion}

Primary immune thrombocytopenia (ITP) remains a diagnosis of exclusion both from nonimmune causes of thrombocytopenia and ITP that develops in the context of other disorders (secondary ITP). [13]. HIT may be suspected in a patient receiving heparin based on a fall in the platelet count. The diagnosis of HIT should initially be judged on clinical grounds. A possible further use of clinical scores is that they may allow the use of more rapid but less sensitive tests to rule out the diagnosis in patients based on pretest probability 4Ts scores (table 1) [14]. 


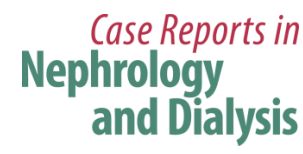

\begin{tabular}{l|l}
\hline \multicolumn{2}{l}{ Case Rep Nephrol Dial 2015;5:152-159 } \\
\hline DOI: 10.1159/000435806 & $\begin{array}{l}\text { C 2015 S. Karger AG, Basel } \\
\text { www.karger.com/cnd }\end{array}$ \\
\hline
\end{tabular}

Al-Jafar et al.: Drug-Immune Thrombocytopenia with Thrombosis versus Heparin-Induced Thrombocytopenia: A Critical Clinical Controversy

The early investigators assumed that DDAbs must be found in patients with DITP [15]. DDAbs react directly with the sensitizing drug itself to form an 'immune complex,' and that complex can react with the target cell, causing DITP, a unique clinical syndrome characterized by severe thrombocytopenia, bleeding, and thrombosis by both DDAbs and immune complex mechanisms $[16,17]$. The antibiotic vancomycin is known to be associated with marked thrombocytopenia and DDAbs [18]. It is also known that continuous infusion of vancomycin through a central venous catheter can be associated with extensive superior vena cava thrombosis [19]. Vancomycin-induced thrombocytopenia may last for many months and, when associated with renal failure, can persist even longer. In a study of vancomycininduced thrombocytopenia, von Drygalski et al. [20] identified vancomycin-dependent antibodies and found that in patients with vancomycin-dependent antibody thrombocytopenia, platelet levels dropped by a mean of $93 \%$ from pretreatment values while they were receiving vancomycin. Vancomycin-dependent antiplatelet antibodies were also detected in patients who received the antibiotic and subsequently developed thrombocytopenia, and the absence of these antibodies in patients who are administered the drug and show stable platelet counts indicates that the vancomycin-dependent antiplatelet antibodies are the cause of the thrombocytopenia [21]. The process of DITP is initiated by an antigen. It is an immune-mediated process that could be carried out by other cross-reacting agents [22, 23].

The HIT test used was Immucor Gamma LIFECODEs PF4 Enhanced assay. It has several limitations. Erroneous results can occur due to bacterial contamination of test materials, inadequate incubation periods, inadequate washing or decanting of test wells, exposure of substrate to stray light, omission of test reagents, exposure to higher or lower than prescribed temperatures, or omission of steps. The Enhanced assay is a qualitative solid-phase enzyme-linked immunosorbent assay (ELISA) designed to detect reactive antibodies. Therefore, the presence of immune complexes or other immunoglobulin aggregates in the patient sample may cause an increase in nonspecific binding and produce false-positive results. Thus, the assay result should not be used as the sole basis for a clinical decision [24]. Furthermore, the diagnosis of HIT based predominantly on HIT laboratory tests may lead to an overdiagnosis. The heparin/PF4 ELISA test has a high rate of false-positive results, particularly in patient populations undergoing coronary artery bypass grafting, in which a positive rate as high as $50 \%$ was detected by postsurgical day 5 [25]. No single assay, however, has $100 \%$ sensitivity and specificity. Although testing becomes most effective when functional and antigen tests are done in combination and multiple samples are taken, this approach is often impractical, and the results are unlikely to be available in a timely manner [26].

The case reported here was a critical clinical challenge and a controversial medical problem; in our opinion it was DITP rather than HIT because HIT test false-positive results can be produced by several factors [24]. Vancomycin, which is the affiancing drug, was repeated several times over the 3 months of severe persistent thrombocytopenia. In the literature, the dug is confirmed to cause prolonged thrombocytopenia especially in patients with end-stage renal failure [19]. This patient developed multiple thrombosis in the absence of heparin while vancomycin related immune complexes known to cause thrombosis [27]. The patient's platelet count did not regenerate even 3 months after stopping heparin, which usually happens within 5-7 days following heparin withdrawal [9]. This patient underwent an uneven course of thrombocytopenia, thrombosis, and continuous bleeding over 3 months before her death. Diagnosis and treatment were difficult because of the similarity between DITP associated with thrombosis and HIT associated with thrombosis. 


\section{Case Reports in \\ Nephrology and Dialysis}

\begin{tabular}{l|l}
\hline Case Rep Nephrol Dial 2015;5:152-159 \\
\hline DOI: 10.1159/000435806 & $\begin{array}{l}\text { ○ 2015 S. Karger AG, Basel } \\
\text { www.karger.com/cnd }\end{array}$ \\
\hline
\end{tabular}

Al-Jafar et al.: Drug-Immune Thrombocytopenia with Thrombosis versus Heparin-Induced Thrombocytopenia: A Critical Clinical Controversy

\section{Conclusion}

Correlating the clinical picture of the thrombocytopenic patient, the drugs used before thrombocytopenia and the result of the HIT test is mandatory for interpreting the diagnosis. Treatment of thrombocytopenia must be undertaken with a heightened awareness and attention to thrombotic events, because other ITPs as well as HIT can be highly thrombogenic. Cross-reactions of DDAbs and HIT antibodies to PF4 can lead to false-positive results even in the confirmatory test; thus, the HIT assay result should not be used as the sole basis for a clinical decision. Although acute thrombocytopenia can spontaneously resolve, it can also lead to extensive morbidity and even mortality. The D-ITP and HIT are controversial medical issues, which need to be researched further. The distinction between the two serious clinical statuses is most important because an erroneous diagnosis will lead to conflicting treatments.

\section{Acknowledgment}

The authors thank Sarfraz Hussain from the Amiri Hospital blood bank and the central blood bank platelet serology laboratory.

\section{Disclosure Statement}

The authors have no conflicts of interest to disclose.

\section{References}

1 Arnold DM, Kukaswadia S, Nazi I, Esmail A, Dewar L, Smith JW, Warkentin TE, Kelton JG: A systematic evaluation of laboratory testing for drug-induced immune thrombocytopenia. J Thromb Haemost 2013;11:169-176.

2 Warkentin TE: Drug-induced immune-mediated thrombocytopenia - from purpura to thrombosis. N Engl J Med 2007;356:891-893.

3 Provan D, Stasi R, Newland AC, Blanchette VS, Bolton-Maggs P, Bussel JB, Chong BH, Cines DB, Gernsheimer TB, Godeau B, Grainger J, Greer I, Hunt BJ, Imbach PA, Lyons G, McMillan R, Rodeghiero F, Sanz MA, Tarantino M, Watson S, Young J, Kuter DJ: International consensus report on the investigation and management of primary immune thrombocytopenia. Blood 2010;115:168-186.

4 Rodeghiero F, Stasi R, Gernsheimer T, Michel M, Provan D, Arnold DM, Bussel JB, Cines DB, Chong BH, Cooper N, Godeau B, Lechner K, Mazzucconi MG, McMillan R, Sanz MA, Imbach P, Blanchette V, Kühne T, Ruggeri M, George JN: Standardization of terminology, definitions and outcome criteria in immune thrombocytopenic purpura of adults and children: report from an international working group. Blood 2009;113:2386-2393.

5 Warkentin TE: Heparin-induced thrombocytopenia: pathogenesis and management. Br J Haematol 2003;121:535-555.

6 DiGiovanni CW: Current concepts review: heparin-induced thrombocytopenia. Foot Ankle Int 2008;29:1158-1167.

7 Ahmed I, Majeed A, Powell R: Heparin induced thrombocytopenia: diagnosis and management update. Postgrad Med J 2007;83:575-582.

8 Cuker A, Cines DB: How I treat heparin-induced thrombocytopenia. Blood 2012;119:2209-2218.

9 Chong BH: Heparin-induced thrombocytopenia. J Thromb Haemost 2003;1:1471-1478.

10 Sarpatwari A, Bennett D, Logie JW, Shukla A, Beach KJ, Newland AC, Sanderson S, Provan D: Thromboembolic events among adult patients with primary immune thrombocytopenia in the United Kingdom General Practice Research Database. Haematologica 2010;95:1167-1175.

11 Thachil J, Callaghan T, Martlew V: Thromboembolic events are not uncommon in patients with Immune thrombocytopenia. Br J Haematol 2010;150:480-497. 


\begin{tabular}{l|l}
\hline \multicolumn{2}{l|}{ Case Rep Nephrol Dial 2015;5:152-159 } \\
\hline DOI: 10.1159/000435806 & $\begin{array}{l}\text { C 2015 S. Karger AG, Basel } \\
\text { www.karger.com/cnd }\end{array}$ \\
\hline
\end{tabular}

Al-Jafar et al.: Drug-Immune Thrombocytopenia with Thrombosis versus Heparin-Induced Thrombocytopenia: A Critical Clinical Controversy

12 Halevy D, Radhakrishnan J, Markowitz G, Appel G: Thrombotic microangiopathies. Crit Care Clin 2002;18:309-320.

13 Cines DB, Liebman H, Stasi R: Pathobiology of secondary immune thrombocytopenia. Semin Hematol 2009;46(Suppl 2):S2-S14.

14 Watson H, Davidson S, Keeling D: Guidelines on the diagnosis and management of heparin-induced thrombocytopenia: second edition. Br J Haematol 2012;159:528-540.

15 Aster RH, Bougie DW: Drug-induced immune thrombocytopenia. N Engl J Med 2007;357:580-587.

16 Curtis BR, Kaliszewski J, Marques MB, Wasif Saif M, Nabelle L, Blank J, McFarland JG, Aster RH: Immunemediated thrombocytopenia resulting from sensitivity to oxaliplatin. Am J Hematol 2006;81:193-198.

17 Meyer T, Robles-Carrillo L, Robson T, Langer F, Desai H, Davila M, Amaya M, Francis JL, Amirkhosravi A: Bevacizumab immune complexes activate platelets and induce thrombosis in FCGR2A transgenic mice. J Thromb Haemost 2009;7:171-181.

18 Mintzer DM, Billet SN, Chmielewski L: Drug-induced hematologic syndromes. Adv Hematol 2009;2009:495863.

19 Leroy S, Piquet P, Chidiac C, Ferry T: Extensive thrombophlebitis with gas associated with continuous infusion of vancomycin through a central venous catheter. BMJ Case Rep 2012;2012:pii: bcr20012006347.

20 Drygalski AV, Curtis BR, Bougie DW, McFarland JG, Ahl S, Limbu I, Baker KR, Aster RH: Vancomycin-induced immune thrombocytopenia. N Engl J Med 2007;356:904-910.

21 Aster RH, Curtis BR, McFarland JG, Bougie DW: Drug-induced immune thrombocytopenia: pathogenesis, diagnosis and management. J Thromb Haemost 2009;7:911-918.

22 Nielsen NH, Linneberg A, Menne T, Madsen F, Frolund L, Dirksen A, et al: Persistence of contact allergy among Danish adults: an 8-year follow-up study. Contact Dermatitis 2001;45:350-353.

23 Baker DC, Brassard J: Review of continuing education course on hemostasis. Toxicol Pathol 2011;39:281288.

24 Immucor GTI Diagnostics Inc.: Lifecodes PF4 Enhanced Assay, 303288 IFUEN REV B. 2013. US patent no.5, 972 and 718

25 Chandra AB, Mittal N, Sambidi S, Belur A, Pathak S, Pathak H, Xu Y: Low incidence of clinically significant heparin induced thrombocytopenia after cardiopulmonary bypass surgery. Transfusion 2013;5.

26 Jang IK, Hursting MJ: When heparins promote thrombosis: review of heparin-induced thrombocytopenia. Circulation 2005;111:2671-2683.

27 Schnuch A, Brasch J, Uter W: Polysensitization and increased susceptibility in contact allergy: a review. Allergy 2008:63:156-167.

The work was done at the AMIRI Hospital, Kuwait City, and the HIT test was performed at the Kuwait Central Blood Bank. 
Al-Jafar et al.: Drug-Immune Thrombocytopenia with Thrombosis versus Heparin-Induced Thrombocytopenia: A Critical Clinical Controversy

Table 1. Pretest probability 4Ts scores

\begin{tabular}{|c|c|c|c|}
\hline & \multicolumn{3}{|l|}{ Points } \\
\hline & 2 & 1 & 0 \\
\hline Thrombocytopenia & $\begin{array}{l}>50 \% \text { fall and platelet } \\
\text { nadir } \geq 20 \times 10^{9} / 1\end{array}$ & $\begin{array}{l}30-50 \% \text { fall or platelet } \\
\text { nadir } 10-19 \times 10^{9} / 1\end{array}$ & $\begin{array}{l}<30 \% \text { fall or platelet } \\
\text { nadir }<10 \times 10^{9} / 1\end{array}$ \\
\hline $\begin{array}{l}\text { Timing of thrombocy- } \\
\text { topenia onset }\end{array}$ & $\begin{array}{l}\text { clear onset between } \\
\text { days } 5 \text { and } 10\end{array}$ & $\begin{array}{l}\text { no clear onset because of } \\
\text { missing platelet count }\end{array}$ & $\begin{array}{l}\text { no recent heparin, but } \\
\text { platelets } \leq 4 \times 10^{9} / 1\end{array}$ \\
\hline Thrombosis & new thrombosis & $\begin{array}{l}\text { progressive or recurrent } \\
\text { thrombosis }\end{array}$ & none \\
\hline $\begin{array}{l}\text { Thrombocytopenia } \\
\text { cause }\end{array}$ & $\begin{array}{l}\text { no cause other than } \\
\text { heparin }\end{array}$ & possible other cause & definite other cause \\
\hline
\end{tabular}

Points were 0,1 , or 2 for each of the four categories for a maximum possible score of 8 . A pretest probability score of 6-8 was considered high, 4-5 intermediate, and 0-3 low. The first day of immunizing heparin exposure is considered day 0 ; the day the platelet count begins to fall is considered the day of onset of thrombocytopenia. 